\title{
Metastatic Prostate Cancer to the Spine and a PSA of 5666: A Case Report
}

\author{
Curtis W. Slipman, MD, Rajeev K. Patel, MD, Evan S. Siegelman, MD, Michael Cirigliano, MD, Atul L. \\ Bhat, MD, Zacharia, Isaac, MD, and David Lenrow, MD
}

Prostate cancer is the most commonly diagnosed cancer and the second most common cause of cancer death among American men. To our knowledge, the highest reported prostate specific antigen (PSA) level on initial presentation is $3280 \mathrm{ng} / \mathrm{mL}(1)$. In this case report, we discuss a 46year-old African-American man with back pain of 1-month's duration. A magnetic resonance imaging study of the lumbar spine revealed numerous osseous metastatic lesions, and the PSA level was found to be $5666 \mathrm{ng} / \mathrm{mL}$. He was treated with oral narcotics and a Duragesic patch to achieve analgesia and bicalutamide (Casodex ${ }^{\circledR}$ ) and leuprolide acetate

A 46-year-old man was referred to this academic spine center with a chief complaint of low back pain. He described a 1-month duration of excruciating low back pain superimposed upon a background of chronic, episodic low back pain. The pain was located across the low lumbar region, with radiation into both anterior thighs. There was no radiation of symptoms distal to the knees. He rated the intensity of his pain as 100/100 on the visual analogue scale. All body positions provoked pain, but none as severe as the pain experienced when lying supine at night. The patient reported complete symptom relief with ibuprofen. A review of systems revealed diminished appetite with subsequent, expected weight loss, and the patient was unable to quantify either the amount or the approximate interval during which his weight loss occurred. There were no other symptoms of metastatic disease present; as the patient denied pain elsewhere, fever, chills, nausea, vomiting, headache, dizziness, vertigo, blurry vision, diplopia, diarrhea, constipation, abdominal discomfort, melena, hematochezia, dysuria, hematuria, frequency, chest pain, shortness of

From the departments of Rehabilitation Medicine, Radiology, and Internal Medicine, University of Pennsylvania, Philadelphia, Pennsylvania and Department of Orthopedics, University of Rochester Medical Center, Rochester, New York. Address correspondence: Curtis W. Slipman, MD, Director, The Penn Spine Center, Ground Floor White Bldg., 3400 Spruce Street, Philadelphia, PA 19104. Email: slipman@mail.med.upenn.edu
(Lupron ${ }^{\circledR}$ ) therapy for androgen blockade. Later in his course, he required chemotherapy due to hormone-refractory prostate cancer. The patient has done well as shown at his latest follow-up at 48 months. The objective of this report is to discuss the first patient with metastatic prostate cancer to the spine with PSA level greater than 3,500 ng/ $\mathrm{mL}$.

Keywords: Spine metastasis, prostate cancer, prostatic specific antigen, magnetic resonance imaging

breath, cough, or hemoptysis.

The past medical history was significant for a prostatic specific antigen (PSA) level of 11 approximately 2 years earlier without any intervening diagnostic or therapeutic interventions. His primary care physician had attempted multiple times through written and telephone communication to arrange for a follow-up visit without success. The family medical history was remarkable for his father's dying of prostate cancer at age 60 and his mother's dying with kidney cancer at age 58. There was also a 30-year pack a day tobacco history.

Physical examination revealed a well-developed, well-nourished African-American man with normal mood and affect; who was alert and oriented to person, place and time. There were no cutaneous lesions, no lymphadenopathy and no spinal deformity. Pulses were 2+ and symmetric; with normal capillary filling at the radial, ulnar and dorsalis pedis arteries. Although there was no extremity atrophy, there was exquisite tenderness to mild pressure application over the low lumbar spine, and pain with pelvic rocking and sustained hip flexion. Root-tension signs were negative. There was mild tightness of the bilateral hamstrings. The patient was able to heel walk and toe walk, and there were no dermatomal or myotomal deficits. Cerebellar function, coordination, and cranial nerves II to XII were intact. A "rock hard prostate" had been noted on recent rectal examination by his internist, and this examination was deferred. There had been no recent radiologic studies per- 
formed during the past 2 years. An immediate magnetic resonance imaging (MRI) of the lumbar spine (Figs. $1 A, B, C)$ was ordered to rule out metastatic spine disease.

The patient was subsequently admitted for aconsultation by urology and oncology. Initial laboratory studies showed chronic anemia, most likely from bone marrow infiltration from metastatic disease. A bone scan demonstrated diffuse osseous metastasis. Eleven single prostate biopsies demonstrated adenocarcinoma. Pathology demonstrated a Gleason score of nine throughout the prostate.

Treatment was initiated with ketoconazole and bicalutamide (Casodex ${ }^{\circledR}$ ) therapy in an effort to create total androgen blockade. Analgesia was achieved with morphine sulfate

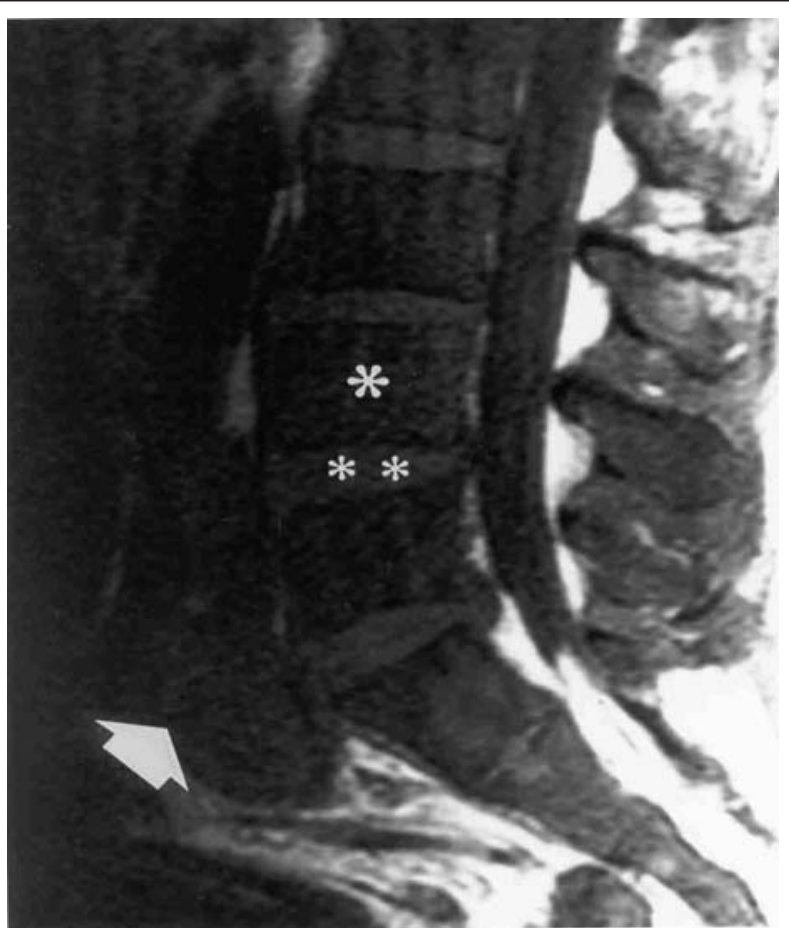

Fig. 1.A. Sagittal T1-weighted spin-echo image (TR = 3D $550 \mathrm{msec}, \mathrm{TE}=3 \mathrm{D} 8 \mathrm{msec}$ ) shows diffuse, heterogenous low-signal intensity marrow suggestive of an infiltrative process ${ }^{(*)}$. On T1-weighted images, bone marrow should have a higher signal intensity than the nucleus pulposus ${ }^{(* *)}$ secondary to the lipid content of the former. The low signal intensity suggests that the marrow lipid is being replaced by some other tissue. Retroperitoneal adenopathy is present in the prevertebral soft tissues (arrows).
Fig. 1.B. Sagittal T2-weighted, fat suppressed, fast spin-echo image $(\mathrm{TR}=3 \mathrm{D} 500 \mathrm{msec}, \mathrm{TE}=$ 3D $9 \mathrm{msec}$ ) shows heterogenous marrow with both low and intermediate signal intensity components. The low-signal-intensity T2-components likely correspond to regions of sclerosis (osteoblastic metastatic disease was confirmed on subsequent radiography).
Fig. 1.C. Axial T1-weighted spin echo image $(\mathrm{TR}=3 \mathrm{D} 500 \mathrm{msec}, \mathrm{TE}=3 \mathrm{D} 9 \mathrm{msec})$ shows the infiltrated marrow ${ }^{(*)}$ and an enlarged mass of iliac adenopathy (arrow). 
(MS-Contin ${ }^{\circledR}$ ) 120 mg by mouth every 8 hours, morphine sulfate (MSIR $\left.{ }^{\circledR}\right) 30 \mathrm{mg}$ by mouth every 4 hours as needed, and a Duragesic patch 50mg/hr changed every 3days. Approximately 2 weeks later he was seen in follow-up by oncology, at which time leuprolide acetate (Lupron ${ }^{\circledR}$ ) therapy was initiated. The patients' PSA level approximately 3 months after initiation of treatment was $<0.2 \mathrm{ng} /$ $\mathrm{mL}$.

Almost 48 months after initial presentation, the patient is doing remarkably well, as demonstrated by his active lifestyle and normal follow-up studies. He continues to work as a professional musician, has gotten married, and has no significant limitations in his lifestyle. He denies any spinal pain and only reports episodic pruritus following chemotherapy administration. His PSA levels are undetectable, and recent bone scans reveal no new osseous lesions. In addition, recent retroperitoneal computed tomography has been remarkable for the absence of lymphadenopathy and an unremarkable prostate and urinary bladder.

\section{DISCUSSION}

Prostate cancer is the most commonly diagnosed cancer and the second most common cause of cancer death among American men. An estimated 179,000 new cases of prostate cancer were diagnosed in 1999, with 37,000 deaths reported. It has decreased over the past several years because of screening. Prostate cancer accounts for $29 \%$ of all newly diagnosed cancer in men in the United States each year and for $13 \%$ of all cancer deaths (2). It is estimated that 1 in 10 American men will develop prostate cancer in his lifetime. The age-adjusted incidence and mortality have been increasing steadily. With the advent of the assay for PSA, an aging population and widespread screening efforts, a $90 \%$ increase in incidence is expected from 1985 to the year 2000 (3). Mortality has likewise increased, from 15,000 in 1965 , to 24,000 in 1983 , to an estimated 41,400 deaths attributed in 1996 (2, 4, 5). A $37 \%$ increase in prostate cancer mortality is estimated by the year 2000 (3).

Prostate cancer incidence and death rates are strikingly different for black and white men in the United States. The age-adjusted incidence is $50 \%$ higher in blacks, and the age-adjusted mortality is more than double the rate found for white men (6). Black men are far more likely to present with a higher stage of disease, although stage-for-stage survival appears to be similar (6-8). These differences persist in higher-income blacks, suggesting that socioeco- nomic factors alone do not account for the discrepancies (9).

The true prevalence of prostate cancer is difficult to estimate, given the high incidence of latent prostate cancer in many autopsy studies. Holund found latent carcinoma in $22 \%$ of 223 consecutive autopsy specimens, including 9\% of men 50 to 59 years old, $12.5 \%$ of men 60 to $69,32 \%$ of men 70 to $79,37 \%$ of men 80 to 89 , and $60 \%$ of men older than $90^{10}$.

There are geographic differences in the incidence and mortality of prostate cancer. Both occur more frequently in North America and Northern Europe than in other areas, and rarely in Asia.

Evidence for a specific inheritance pattern in prostate cancer was lacking until recently. Carter and associates (11) performed segregation analysis on family history collected from 691 prostate cancer patients; they postulated a Mendelian autosomal dominant inheritance model. This rare allele was highly penetrant, in that $88 \%$ of the carriers were estimated to have prostate cancer by the age of 85 , compared to $5 \%$ of noncarriers. The autosomal dominant allele accounted for $43 \%$ of the cases diagnosed before the age of 56, but only $9 \%$ of prostate cancers diagnosed before the age of 85 .

Prostate-specific antigen was described initially by Wang et al. in 1979 (12). This organ-specific protein was first discovered by Hara et al. in 1971 (13). Prostate specific antigen is a glycoprotein produced exclusively by the epithelial cells lining the prostatic ducts. It is secreted directly into the lumen, where it performs its primary function of liquefying the seminal coagulum $(13,14)$. As expected, it is found in high concentrations in the seminal plasma. Papsidero et al identified PSA in human serum and proved it to be identical to that found in prostate tissue (15). Due to the high specificity for prostate tissue, PSA has displaced acid phosphatase as the primary serum tumor marker for prostate cancer. The most common method of measuring serum is the Tandem- $\mathrm{R}$ (Hybritech) assay (16, 17). This radioimmunoassay uses two murine monoclonal antibodies and has a normal range of 0 to $4.0 \mathrm{ng} / \mathrm{mL}$.

Since PSA is prostate-tissue-specific and not prostate-cancer-specific, benign conditions of the prostate gland can result in abnormal elevation. False elevations of PSA can occur after urethral instrumentation, prostate biopsy, urinary retention, and prostatitis $(18,19)$. Benign prostatic hyperplasia (BPH) is the most common benign disease of 
the prostate to cause an abnormal elevation of serum PSA. The prevalence of BPH in men at risk for prostate cancer ( $>50$ years) is high, therefore limiting the usefulness of an abnormal serum PSA alone to diagnose prostate cancer.

However, there are many studies that demonstrate a significantly higher serum PSA in patients with prostate cancer than in those with BPH. Oesterling combined three large studies and found that the diagnostic accuracy of PSA alone is $65 \%$ for organ-confined disease when the serum level is greater than $4.0 \mathrm{ng} / \mathrm{mL}$ and $70 \%$ when it is greater than $10.0 \mathrm{ng} / \mathrm{mL}$ (10). The positive predictive values are calculated at $49 \%$ and $75 \%$, respectively. It has been demonstrated that, in the presence of cancer, serum PSA levels increase at a rate proportional to the volume of intracapsular tumor, an average of $3.5 \mathrm{ng} / \mathrm{mL} / \mathrm{g}$ of intracapsular tumor regardless of extracapsular spread(20).

Investigators have examined the relationship between serum PSA levels and volume of disease, capsular penetration, and lymph-node metastasis (21). In a study of 301 patients, a PSA level of less than $10 \mathrm{ng} / \mathrm{mL}$ was associated with a $0 \%$ incidence of lymph-node involvement, less than $1 \%$ incidence of tumor volume greater than $12 \mathrm{cc}, 5 \%$ incidence of seminal vesicle involvement, and an $11 \%$ incidence of invasion into the periprostatic fat. When compared to a serum PSA of greater than $50 \mathrm{ng} / \mathrm{mL}$, there was a $45 \%$ incidence of lymph-node involvement, $64 \%$ incidence of tumor volume of greater than 12 cc, $73 \%$ incidence of seminal vesicle invasion, and 55\% incidence of periprostatic fat invasion. These studies serve to illustrate that predictions of disease extent can be made with relative confidence when the PSA level is markedly elevated (50 ng/mL or higher).

Interestingly, tumor volume has been shown to be a more reliable predictor of extracapsular tumor growth. In a study of 176 radical prostatectomy specimens, capsular penetration was found in only $7 \%$ of tumors less than $4 \mathrm{cc}$ in volume but in $86 \%$ of tumors over 12 cc in volume(12). Similarly, Villers et al. reported seminal vesicle invasion in 6\% of tumors less than $4 \mathrm{cc}$ in volume versus $82 \%$ for lesions greater than $12 \mathrm{cc}$ in volume(22).

Recent studies have demonstrated that a serum PSA of greater than $100 \mathrm{ng} / \mathrm{mL}$ yielded a $50 \%$ to $100 \%$ positive predictive value for bone metastasis (23-25). It also demonstrated a negative predictive value of 96 to $99.7 \%$ for patients with a PSA $<10 \mathrm{ng} / \mathrm{mL}(24-26)$. Some data suggest that the annual rate of change in serum PSA is more important than the specific value. Increases of greater than
$0.7 \mathrm{ng} / \mathrm{mL} /$ year have been said to be suggestive of cancer. To our knowledge, the highest reported PSA in the literature prior to this report was $3280 \mathrm{ng} / \mathrm{mL}$, and this level was measured by the Tandem-R assay (1).

Bone scan has traditionally been the gold-standard screening tool for metastasis to bone and has demonstrated a high sensitivity for the presence of bony metastasis, with reports varying from $90 \%$ to $100 \%(27,28)$. However, it is generally accepted that bone scans are nonspecific, and that MRI is the radiologic study of choice to identify osseous metastasis, including the spine (29). Recently, MRI has proven to be an extremely useful modality in the diagnosis of metastatic prostate cancer to bone, especially when other radiographic studies are inconclusive, spinal cord compression is suspected, or determination of response to treatment is needed $(29,30)$. One study showed that, of eight positive bone scans, two were interpreted as negative on MRI; of five equivocal bone scans, one was read as positive on MRI; in addition, serial MRI on six patients with bony metastasis and hormonal treatment demonstrated radiographic and clinical improvement(30). Another study noted that of 19 positive bone scans, MRI was read as negative in 1 ; of 8 equivocal scans, MRI was read as positive in 2. Furthermore, MRI demonstrated additional metastatic lesions that were not shown on bone scan and clearly indicated the areas of spinal cord compression in the five patients with associated myelopathy(31). Magnetic resonance imaging may have a higher diagnostic accuracy than radionuclide bone scan in the evaluation of osseous metastatic disease from prostate cancer. Magnetic resonance imaging can minimize the frequency of false-positive diagnosis of metastatic disease from Paget's disease, benign compression fractures and osteoarthrosis. In addition, it can detect metastatic deposits not present on bone scan images. In comparison to bone scan, Magnetic resonance imaging can image nonosseous structures, such as pelvic and retroperitoneal lymph nodes. With bone scan, adenopathy can only be inferred if there is resultant hydronephrosis. MRI is the radiologic study of choice in detection of metastasis to the spine, especially when plain films and bone scans are negative or equivocal (29). In this case, MRI unequivocally demonstrated the diagnosis of diffuse metastatic disease to the spine.

\section{CONCLUSION}

This case study of a patient with metastatic prostate cancer to the spine reports the highest PSA level published to date. 


\section{REFERENCES}

1. Vaidya HC, Wolf BA, Garett N et al. Extremely high values of prostate specific antigen in patients with adenocarcinoma of the prostate; Demonstration of the "hook effect.” Clin Chem 1998 34: 2175-2177.

2. Landis, SH, Murray T, Bolden S et al. Cancer statistics, CA 1999; 49:8-31.

3. Coffey DS. Prostate cancer: An overview of an increasing dilemma. Cancer 1993;71 (suppl):880.

4. Silverberg E, Lubera JA. A review of American Cancer Society estimates of cancer cases and deaths. Cancer 1983;33:2.

5. Wynder EL, Mabuchi K, Whitmore WF. Epidemiology of cancer of the prostate. Cancer 1971;28:344.

6. Mebane C, Gibbs T, Horm J. Current status of prostate cancer in North American black males. J Natl Med Assoc 1990;82:782.

7. Polednak AP, Flannery JT. Black versus white racial differences in clinical stage at diagnosis and treatment of prostate cancer in Connecticutt. Cancer 1993 70:2152.

8. Targonski PV, Guinan P, Phillips CW. Prostate cancer: The stage disadvantage in the black male. J Natl Med Assoc 1990;83:1094.

9. Polednak AP. Cancer mortality in a higher-income black population in New York State: Comparison with rates in the United States as a whole. Cancer 1990; 66:1654.

10. Holund B. Latent prostatic cancer in a consecutive autopsy series. Scand J Urol Nephrol 1980;14:29.

11. Carter BS, Beaty TH, Steinberg GD et al. Mendelian inheritance of familial prostate cancer, Proc Natl Acad Sci USA 1992;89:3367.

12. Hara M, Inore T, Fukuyama T. Some physio-chemical characteristics of gamma-seminoprotein, an antigenic compound specific for human seminal plasma. Jpn $J$ Legal Med 1971;25:322

13. Lilja, H, Lourell CB. The predominant protein in human seminal coagulate. Scand J Clin Lab Invest 1985;45:635.

14. Lilja, H. A kallikrein-like serine protease in prostate fluid leaves the predominant seminal vesicle protein. J Clin Invest 1985;76:1899.

15. Papsidero LD, Wang MC, Valenzuela LA et al. Aprostate antigen in sera of prostatic cancer patients. Cancer Res 1980;40:2428.

16. Myrtle JF, Klimley PG, Ivor LP et al. Clinical utility of prostate-specific antigen (PSA) in the management of prostate cancer (abstract). Tumour Biol 1987;8:353.

17. Yang, N. Pros-Check PSA. A double antibody radioimmunoassay for prostate specific antigen. In Catalona WJ, Coffey, DS, Karr JP (eds). Clinical aspects of prostate cancer. New York, Elsevier, 1989;pp172-178.

18. Seamonds, B, Yang N, Anderson K et al. Evaluation of prostate-specific antigen and prostatic acid phosphatase as prostate cancer markers. Urology 1986 28:472.

19. Stamey TA. Prostate specific antigen in the diagnosis and treatment of adenocarcinoma of the prostate. Monogr Urol 1989;10:49.

20. Villers AA, McNeal JE, Redwine EA et al. Pathogenesis and biological significance of seminal vesicle invasion in prostatic adenocarcinoma. J Urol 1990;143:1183.

21. Lange, PH, Ercole CJ, Lightner DJ et al. The value of serum prostate specific antigen before and after radical prostatectomy. J Urol 1989;141:873.

22. McNeal JE, Villers AA, Redwine EA et al. Capsular penetration in prostate cancer. Significance for natural history and treatment. Am J Surg Pathol 1990;14:240.

23. Rana A, Karamanis K, Lucal MG et al: Identification of metastatic disease by T category, Gleason score and serum PSA level in patients with carcinoma of the prostate. Br J Urol 1992;69:277-81.

24. Wolff JM, Bares R, Jung PK et al. Prostate-specific antigen as a marker of bone metastasis in patients with prostate cancer. Urol Int 1996;56:169-173.

25. Wolff JM, Zimny M, Borchers H, Wildberger J, Buell $\mathrm{U}$, Jakse G. Is prostate-specific antigen a reliable marker of bone metastasis in patients with newly diagnosed cancer of the prostate? Euro Urol 1998;33, 376-381.

26. Oesterling JE. Prostate-specific antigen: A critical assessment of the most useful tumour marker for adenocarcinoma of the prostate. J Urol 1991;145:907-923. Corrie D, Timmons JH, Bauman JM et al. Efficacy of follow-up bone scans in carcinoma of the prostate. Cancer 1988 61:2453-2454.

28. Wahner HW, Brwon ML. Role of bone scanning. In SIM FH (ed). Diagnosis and Management of Metastatic Bone Disease. New York, Raven Press, 1988, pp. 51-67.

29. Fuen LG, Savaraj N. Detection of occult bone metastasis by MRI scan. J Fla Med Assoc 1990; 77:881883.

30. Turner JW, Hawes DR, Williams RD. Magnetic resonance imaging for the detection of prostate cancer metastatic to bone. J Urol 1993; 149:1482-1484.

31. Fujii Y, Higashi Y, Owada F et al. Magnetic resonance imaging for the diagnosis of prostate cancer metastatic to bone. Brit J Urol 1995; 75:54-58. 